\title{
Factors affecting the loss of MED12-mutated leiomyoma cells during in vitro growth
}

\author{
Jeannine Bloch ${ }^{1}$, Carsten Holzmann ${ }^{1}$, Dirk Koczan ${ }^{2}$, Burkhard Maria Helmke ${ }^{3}$, Jörn \\ Bullerdiek ${ }^{1,4}$ \\ ${ }^{1}$ Institute of Medical Genetics, University Rostock Medical Center, D-18057 Rostock, Germany \\ ${ }^{2}$ Institute of Immunology, University Rostock Medical Center, D-18057 Rostock, Germany \\ ${ }^{3}$ Institute of Pathology, Elbe Kliniken, Klinikum Stade, D-21682 Stade, Germany \\ ${ }^{4}$ Center of Human Genetics, University of Bremen, D-28359 Bremen, Germany
}

Correspondence to: Jörn Bullerdiek, email: joern.bullerdiek@med.uni-rostock.de

Keywords: MED12 mutations, uterine leiomyomas, in vitro growth, model system, cell culture

Received: July 28, 2016 Accepted: March 08, $2017 \quad$ Published: March 30, 2017

Copyright: Bloch et al. This is an open-access article distributed under the terms of the Creative Commons Attribution License (CC-BY), which permits unrestricted use, distribution, and reproduction in any medium, provided the original author and source are credited.

\section{ABSTRACT}

Uterine leiomyomas (UL) are the most prevalent symptomatic human tumors at all and somatic mutations of the gene encoding mediator subcomplex 12 (MED12) constitute the most frequent driver mutations in UL. Recently, a rapid loss of mutated cells during in vitro growth of UL-derived cell cultures was reported, resulting in doubts about the benefits of UL-derived cell cultures. To evaluate if the rapid loss of MED12-mutated cells in UL cell cultures depends on in vitro passaging, we set up cell cultures from nine UL from 40-50 year old Caucasian patients with at least one UL. Cultured UL cells were investigated for loss of MED12-mutated cells. Genetic characterization of native tumor samples and adjacent myometrium was done by array analysis. "Aged" primary cultures without passaging were compared to cells of three subsequent passages. Comparative analyses of the mutated/non-mutated ratios between native tissue, primary cells, and cultured tumor cells revealed a clear decrease of MED12-mutated cells. None of the tumors showed gross alterations of the array profiles, excluding the presence of gross genomic imbalances besides the MED12 mutations as a reason for the intertumoral variation in the loss of MED12mutated cells. Albeit at a lesser rate, loss of MED12-mutated cells from cell cultures of UL occurs even without passaging thus indicating the requirement of soluble factors or matrix components lacking in vitro. Identification of these factors can help to understand the mechanisms of the growth of the most frequent type of uterine leiomyomas and to decipher novel drug targets.

\section{INTRODUCTION}

Uterine leiomyomas (UL) are the most prevalent symptomatic human tumors at all constituting a major public health problem $[1,2]$.

Nevertheless, there is a surprising gap of knowledge on the pathobiology of these frequent tumors. Current data strongly suggest that particular somatic mutations of the gene encoding mediator subcomplex 12 (MED12) constitute the most frequent driver mutations in UL [3-7]. Mediator subcomplex 12 is a protein involved in the organisation of the transcription machinery showing a high degree of evolutionary conservation among mammalian species in particular in the region encoded by the UL-hotspot region [8]. While UL-derived cell cultures are a wide-spread model system for research into the pathogenesis and behavior of the tumors [2, 9-11] recent results challenge the usefulness of this model for the group of tumors carrying MED12 mutations. Contrary to the expectations a rapid loss of mutated cells accompanied in vitro growth of UL-derived cell cultures [12]. As a rule, the mutations were not even detectable after early passages of in vitro growth resulting from so far unknown mechanisms. One possible explanation is a decreased growth potential resulting from in vitro passaging. However, an in depth understanding of the 
mechanisms leading to the disappearance of tumor cells in vitro may help to uncover basic growth requirements of MED12-mutated UL and as well as novel drug targets. Thus, it is of pivotal interest to see if the disappearance of mutated cells is a phenomenon linked to enzymatic treatment of cells associated with passaging or occurs independent of this artificial procedure. Accordingly, we have performed a series of experiments aimed at a comparison between cells of UL subjected to repeated passaging and those that were kept in continuous stationary primary culture.

\section{RESULTS}

\section{MED12 mutations were found in the majority of tumors initially analyzed}

Initially, cell cultures from nine UL derived from five patients were set up for cell culture. The results of MED12 mutation analyses revealed mutations of that gene in seven of these tumors (Table 1). Histologically, all seven tumors were typical UL. Of these one carried a $M E D 12$ in-frame deletion and the others showed singlebase substitutions (Supplementary Figure 1). All mutations detected were identical to those previously detected in UL and heterozygous. Accordingly, Sanger sequencing resulted in peaks corresponding to the non-mutated and mutated alleles, respectively. For further evaluation it was presumed that the "non-mutated peak" results from the normal allele of the tumor cells as well as from the two normal alleles of bystander cells not belonging to the tumor cell population. Vice versa, the "mutated peak" is considered to have originated exclusively from the mutated allele of the tumor cells.

\section{In most cases, a decrease of mutated cells was noted even in the primary culture}

First, six of these cases (UL 06/1, UL 06/2, UL 07/1, UL 07/2, UL 12/1, and UL 12/2) were used for a comparative analysis of the peak ratios between native tissue and primary cultures. In case UL 07/3, cells of the primary culture were not available for analysis. Compared to the native tissue the relative height of peaks corresponding to the mutated allele had decreased in the primary cultures of 5/6 tumors (UL 06/1, UL 07/1, UL 07/2, UL 12/1, and UL 12/2) investigated. Nevertheless, a strong variation of the decrease was noted that ranged between an almost complete disappearance of the mutated peak seen in UL $07 / 1$, a huge loss of $67 \%$ to $77 \%$ in UL $07 / 2$ and UL $12 / 2$, and a slight decrease of only $17 \%$ to $27 \%$ observed in UL $06 / 1$ and UL $12 / 1$, respectively (Figure 1 and Supplementary Figure 3). In the remaining case (UL 06/2), a moderate increase of the ratio was noted in the primary culture.

\section{Further disappearance of mutated cells does not require passaging but also occurred in "aging" primary cultures}

As a next step, we were interested in monitoring changes of the frequency of the MED12 mutated cells during in vitro propagation. Therefore, we passaged the "young" primary cultures of five tumors (UL 06/1, UL 06/2, UL 07/1, UL 07/2, and UL 07/3) three times followed by sequencing. In all cases continuous passaging was accompanied by a further reduction of the peak ratio compared to the native tissue $(5 / 5$ cases $)$ as well as to the primary culture ( $4 / 4$ cases, no data of the primary culture were available for UL 07/3) (Figure 2). In the first passages the decline ranged between $60 \%$ and $80 \%$ compared to the corresponding primary culture. In UL 06/1, UL 06/2 and UL $07 / 2$ the mutation was clearly detectable in the first passage but rapidly disappeared in passage 2 and 3 . In contrast, UL 07/1 showed an almost complete loss of the mutated peak already in the first passage. As to further passaging the MED12 mutation was almost undetectable in three of the cases whereas it remained clearly visible until passage 3 in UL 06/2. Moreover, the number of passages where mutated cells were still detectable seems to depend on their frequency in the primary culture.

Next we were interested to see if a simple "aging" of the confluent primary cultures leads to a decrease of the mutated cells. Accordingly, primary cultures of six tumors were kept unpassaged after they had reached confluency. During that time, medium was changed every 2 nd or 3 rd day until they were harvested. Compared to the "young" primary cultures "aging" of the primary culture resulted in a reduced mutated/non-mutated peak ratio in all six tumors (UL 06/1, UL 06/2, UL 07/1, UL 07/2, UL 12/1, and UL 12/2) (Figure 3 and Supplementary Figure 4). In two tumors (UL 12/1 and UL 12/2) "aging" primary cultures were harvested and analyzed at two different times of in vitro growth. The cells were harvested one and two weeks after "young" primary cultures. In general, both points in time revealed a decline in mutated to nonmutated peak compared to the "young" primary cultures. While in UL $12 / 1$ a loss of $60 \%$ and $80 \%$, respectively, was noted in both "aging" primary cultures, in UL $12 / 2$ the ratio of mutated/non-mutated peaks was decreased to $30 \%$ and remained nearly unchanged. However, in neither of the cultures the disappearance of mutated cells was accompanied by clear changes of cellular morphology.

\section{Passaging of the cells accelerated the loss of mutated cells}

We hypothesized that the procedures necessary for passaging did not cause but may accelerate the loss of mutated cells from the cultures. To validate this assumption we have compared the loss in "aging" primary cultures with 
Table 1: Type of $M E D 12$ mutation in leiomyomas with $M E D 12$ mutations included in the study

\begin{tabular}{cccc}
\hline tumors investigated & age & tumor size $\mathbf{( c m )}$ & MED12 status \\
\hline $06 / 1$ & 52 & 7.5 & c.131G $>\mathrm{C}$ \\
$06 / 2$ & & 4.5 & c.131G $>\mathrm{C}$ \\
$07 / 1$ & 47 & 1.8 & c.131G $>\mathrm{A}$ \\
$07 / 2$ & & 1.5 & c.131G $>\mathrm{A}$ \\
$07 / 3$ & & 1.0 & c.126_137del12 \\
$12 / 1$ & 53 & 1.0 & c. $107 \mathrm{~T}>\mathrm{G}$ \\
$12 / 2$ & & 0.6 & c.131G $>\mathrm{A}$ \\
\hline
\end{tabular}

the corresponding cell cultures that had been passaged within the same interval of time. For this comparison, five pairs of 3rd passage cells along with their matching unpassaged primary cultures of the same "age" were used. The results show that in all five cell cultures investigated the loss occurred indeed more rapidly when cells are passaged. While the loss strongly varied between the tumors, in all these pairs the amount of mutated cells in the "aged" cultures exceeded that seen in the $3^{\text {rd }}$ passage cells of the same "age" (Figure 4). Furthermore, in $3 / 4$ cultures the ratio in the "aged" cultures exceeded that observed in the cells of the 1st passage.

\section{The different rates of loss of mutated cells did not correlate with the presence of genomic imbalances in addition to the $M E D 12$ driver mutations}

While the decrease of mutated cells in vitro strongly varied among the different tumors investigated no obvious explanation for these differences was apparent. MED12 mutations in UL are known to coincide often with other genetic aberrations as e.g. deletions of part of the long arm of chromosome 7, rearrangements of the HMGAl locus at 6 p21 or trisomy 12 in a considerable percentage of cases [4]. These alterations might influence the "in vitro behavior" of MED12-mutated cells. Thus, aimed at the question whether or not the differences are due to secondary genomic gains and losses in addition to the MED12 mutations we used CNV arrays for the analysis of the native tumor samples along with their matching myometrium on four UL (UL 06/1, UL 06/2, UL 07/1, and UL $07 / 2$ ) with $M E D 12$ mutations. In none of the tumors investigated relevant alterations of the $\mathrm{CNV}$ profiles became apparent when comparing the leiomyomas with the corresponding normal tissue (Supplementary Figure 6). Thus, the reasons for the different kinetics of the loss of mutated cells still remain to be elucidated.

\section{Loss of mutated cells from the "aging" culture correlates with a detachment of cells from the monolayer}

As to the loss of MED12-mutated cells it can be assumed that the mutated cells become detached from the surface of the culture vessels. Thus, one would expect a high amount of mutated cells in the supernatant. To address this question, we have isolated and sequenced DNA from cells in the supernatant of unpassaged cultures of two UL (UL 12/1 and UL 12/2) and compared with their matching adherent cells. The results confirmed the assumption that mutated cells are lost from the monolayer by their detachment since the amount of mutated cells in the supernatant clearly exceeded that found in the corresponding monolayer (Figure 5 and Supplementary Figure 5). Simultaneously, we analyzed four UL (UL 06/1, UL 06/2, UL 07/1, and UL 07/2) and compared the DNA from the cells in the passages with their matching cells in the supernatants directly obtained after passaging. Consistent with our experience a huge amount of mutated cells was observed in the supernatant of the first passage (Figure 6). Increasing the number of passages revealed a decline of the "mutated" peak in the corresponding supernatant which, however, always remained higher than in the correlating passaged cells. Even if no evidence of mutated cells in the passaged culture was noted, they were still detectable in the corresponding supernatant. The results indicate that the mutated cells are also lost from the cell cultures due to their problems of re-attachment after passaging.

\section{DISCUSSION}

Despite novel approaches to shrink UL and to improve tumor related bleeding $[13,14]$ still unmet medical needs remain in the treatment of these tumors. Factors influencing the growth of UL are of high interest and often have been studied using cells grown in vitro (e.g. see [15-17]) making in vitro cultures derived from leiomyomas a widespread model system for research into biology and treatment of UL (for review see [18]).

As to their molecular pathogenesis, a number of recurrent genetic alterations confined to the ULs but not present in their surrounding myometrial tissue have been identified. For example, certain types of chromosomal deletions and translocations are often detected in UL. Among these, translocations involving chromosomal bands 12q14-15 and deletions of part of the long arm of chromosome 7 are most frequently seen and in the 


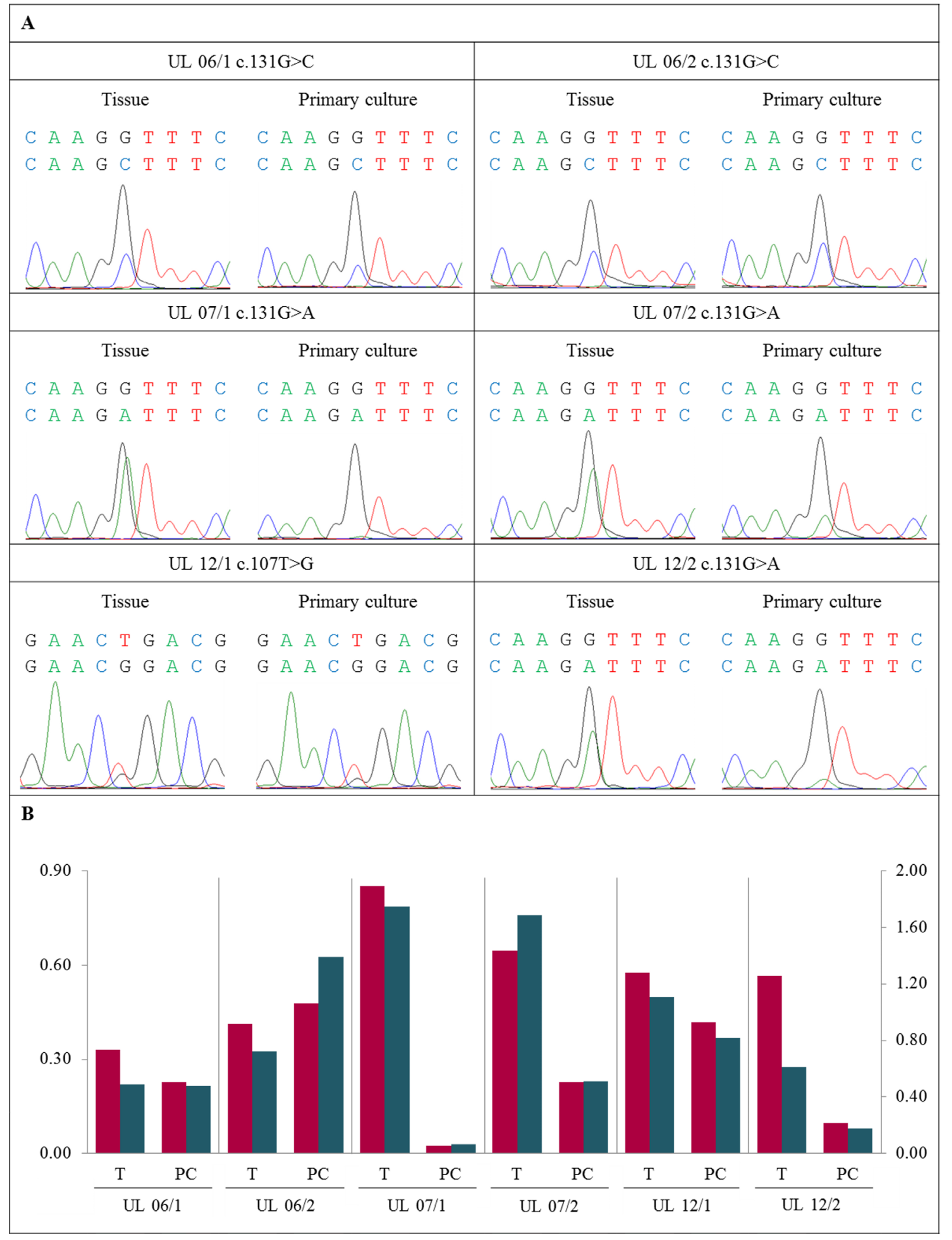

Figure 1: Decrease of $M E D 12-m u t a t e d$ cells in primary cultures compared to native tissue. (A) DNA forward sequence of three codons around the MED12 point mutation of the native tumor tissue (T) and the corresponding primary culture (PC) displaying heterozygous mutations c.131G $>$ C, c.131G $>$ A or c. $107 \mathrm{~T}>\mathrm{G}$, respectively. In UL 06/1, UL 07/1, UL 07/2, UL 12/1 and UL 12/2 a decline of the peak indicating the mutated allele was detected in PC. In case UL 06/2 a moderate increase of the mutated peak was noted. (B) Quantification of mutated to non-mutated allele of MED12 mutation mainly shows a decrease of MED12-mutated cells in PC compared to corresponding T. The quotients of forward sequencing direction (red columns) were shown on primary axis and of reverse (blue columns) on secondary axis. 
former case the gene encoding HMGA2, a member of the family of high mobility group proteins, has been identified as the molecular target of the alteration which becomes drastically up-regulated due to the chromosomal rearrangement [19]. Nevertheless, the majority of UL do not show any chromosomal alterations but mutations of the gene encoding Mediator subcomplex 12 (MED12), a protein involved in the organization of the transcription machinery. In most of these cases only a singly base exchange can be detected but small in-frame deletions and duplications do occur as well. This latter type of mutation occasionally can co-exist with chromosomal alterations except for those targeting HMGA2 [3-7] and clearly represents the most common type of driver mutations in UL. In vitro cells with this type of driver mutation recently were shown to coincide with a strictly reduced growth capacity in vitro, leading to their rapid disappearance in cell culture $[12,20]$. In contrast, other cells from the samples not carrying these mutations of presumed non-tumorigenic origin as well as from tumors carrying $H M G A 2$ rearrangements can survive much longer [12, 20]. The loss of MED12-mutated cells even during early phases of in vitro culturing is a phenomenon challenging many previous data obtained using cell cultures as a model system for research into the biology of UL. While it is reasonable to postulate that only the mutated cells represent the tumor cell population, the mechanisms underlying the observed loss of tumor cells are not understood yet. On the other hand, the loss of tumor cells, at least in part, may explain the rapid loss of estrogen receptors and, albeit at a lower rate, loss of progesterone receptors, repeatedly described for UL cultures based on a decrease of mRNA as well as proteins $[9,21]$. This loss may, at least in part, result from the loss of positive cells from the cultures rather than from the transcriptional downregulation of the corresponding genes as previously assumed. Also, it may explain the gradual loss of cells with some types of cytogenetic alterations as e.g. deletions of the long arm of chromosome 7 known not rarely to co-exist with $M E D 12$ mutations which has been

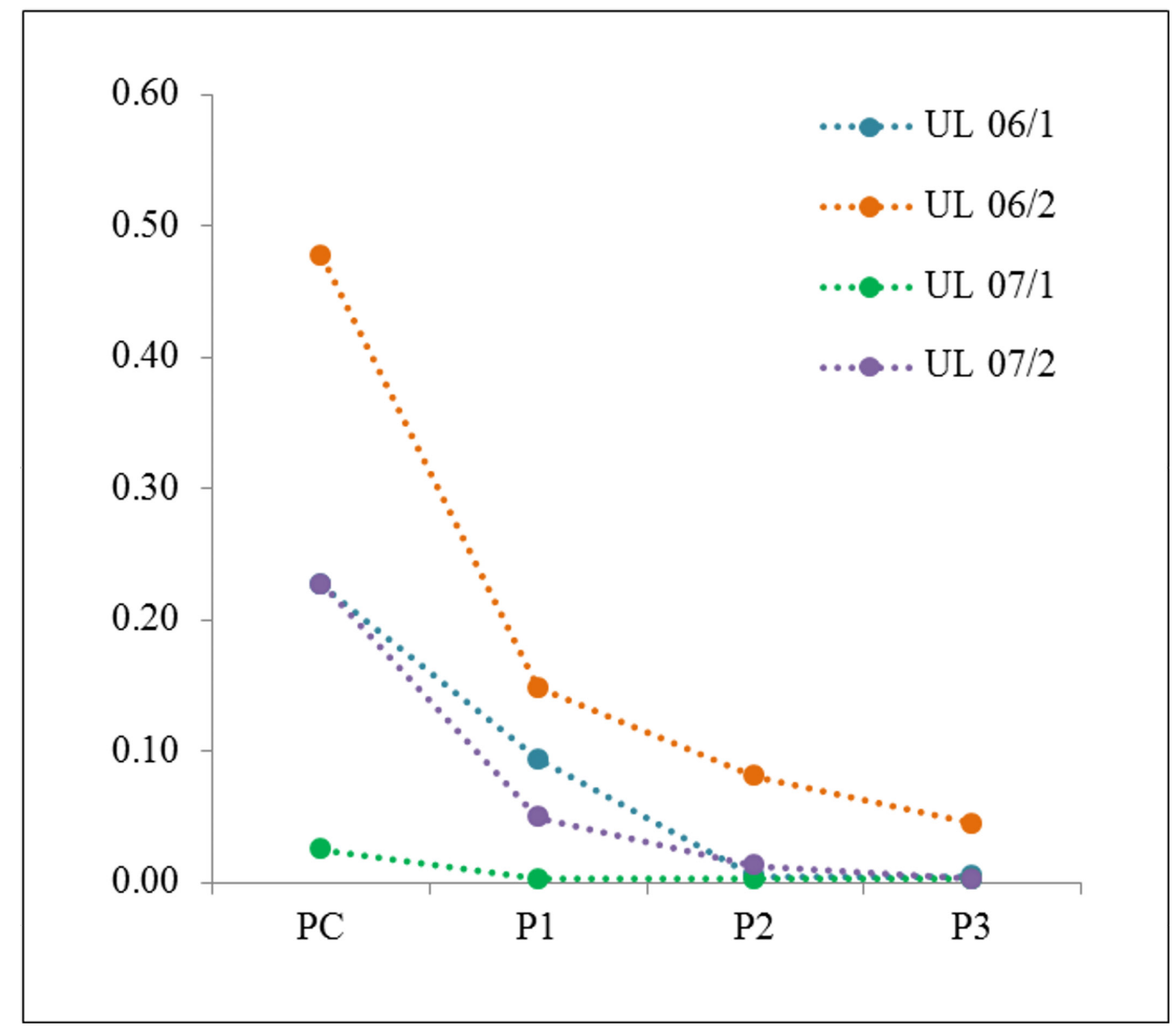

Figure 2: Disappearance of cells with MED12 mutation during in vitro passaging. In all UL investigated a loss of mutated/ non-mutated peak ratio was noted in the first passage (P1) compared to the corresponding primary culture (PC). In the following passages (P2 or P3) the loss further declines to an almost complete loss of mutated cells, except UL 06/2. Data resulting from DNA forward sequencing. 


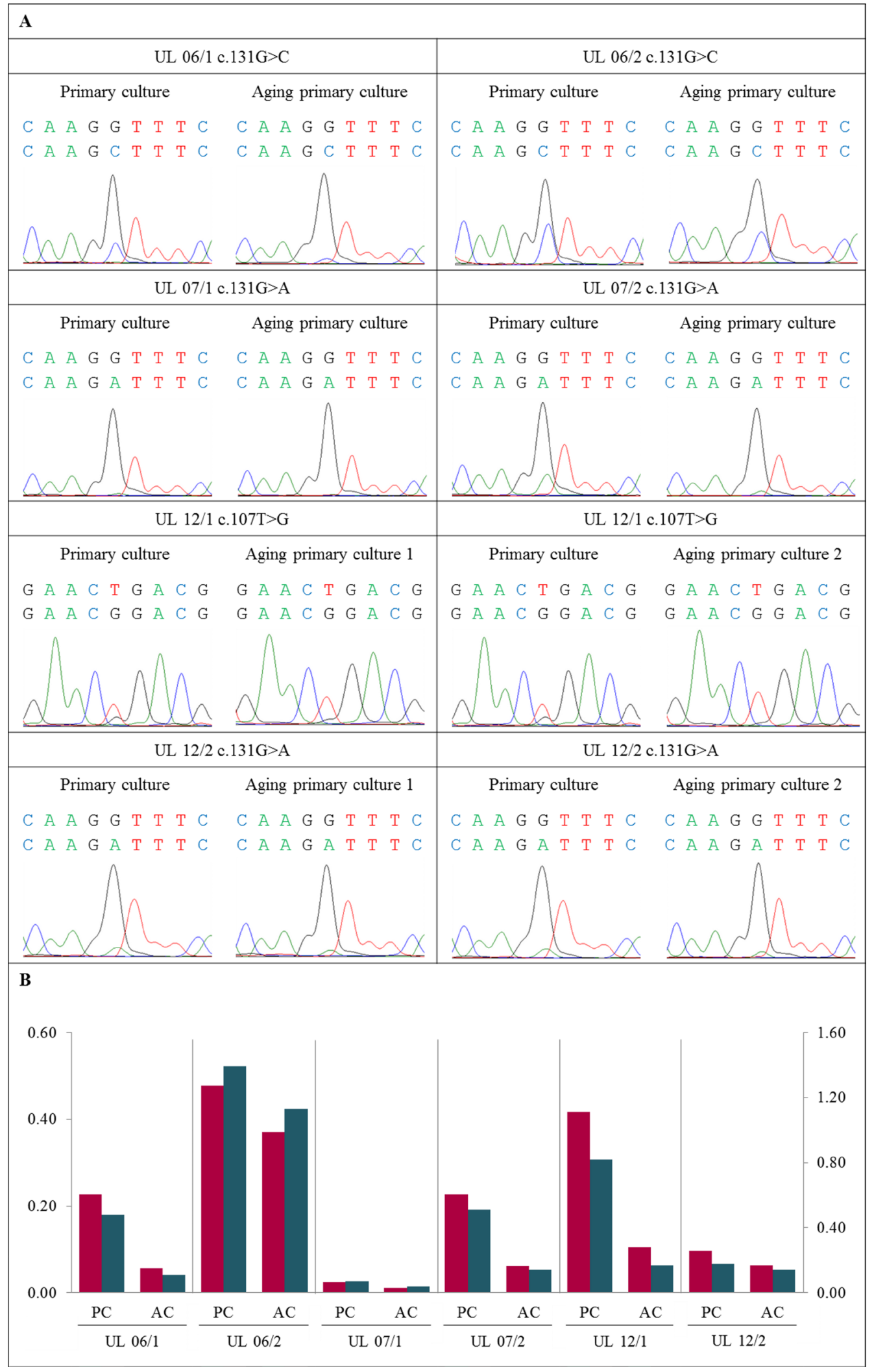

Figure 3: Further disappearance of MED12-mutated cells also occurred in "aging" primary cultures. (A) DNA forward sequence of three codons around the MED12 point mutation of the primary cell culture (PC) and the corresponding "aging" primary cultures (AC) displaying a heterozygous mutation c. $131 \mathrm{G}>\mathrm{C}$, c. $131 \mathrm{G}>\mathrm{A}$ or c. $107 \mathrm{~T}>\mathrm{G}$. In all UL investigated a decline of the "mutated peak" was detected in AC compared to matching PC. (B) Quantification of mutated to non-mutated allele of MED12 mutation shows a decrease of MED 12-mutated alleles in AC compared to corresponding PC. The quotients of forward sequencing direction (red) were shown on primary axis and of reverse (blue) on secondary axis. 
observed in cultures obtained from UL (Sabine Bartnitzke, personal communication).

Herein, we were able to show that the treatment of cells during passaging, while accelerating their loss, does not constitute the main reason for disappearing of the mutated cells because the loss even occurs from stationary "aging" cultures. There is no obvious explanation for the strongly varying rate of loss in vitro but among other possible reasons the type of MED12 mutation may play a role. Nevertheless, also in general there is no explanation for the loss of mutated cells but from our data presented herein it can be concluded that comparably "simple" explanations like e.g. a higher sensitivity of the tumor cells against trypsin treatment do not apply. Basically, two groups of explanations seem plausible.

First, it is reasonable to assume that certain soluble environmental factors not available under in vitro conditions used for the cell cultures are required for the survival of the tumor cells. Among these critical factors lacking in vitro may be growth factors and hormones. For future studies it may be worth to re-address this question by analyzing the decrease of MED12-mutated cells depending on different culture media including those supplemented with hormones.

Secondly, the in vitro growth could depend on rather "physical" factors as e.g. the presence of extracellular matrix (ECM). Interestingly, the relevance of ECM in the growth of UL had been a matter of numerous studies and therapeutical approaches targeting ECM have been proposed repeatedly (e.g. [22-25]). Accordingly, future studies should address the possible influence of ECM presented in vitro and of three-dimensional cell cultures on the disappearance of MED12-mutated cells.

As to the results of the present study we cannot decide if the main reason for the disappearance of the tumor cells is the lack of an appropriate matrix or of growth factors supporting cellular proliferation. On the other hand, growth of non MED12-mutated bystander cells does clearly not depend on these requirements because a rapid overgrowth of these latter cells can be noted. In general, the relative amount of non-mutated cells likely indicates that these cells are not solely derived from the tumor vasculature but represent a considerable amount of the "leiomyoma-stroma" as well. Cell cultures thus represent an interesting source to investigate the growth balance between tumor cells and non-tumorigenic cells and its disturbances, respectively because they provide an interesting system to identify factors that selectively support the growth of tumor cells. In turn, these factors may be of relevance for in vivo growth thus representing possible therapeutic targets. Furthermore, threedimensional cell cultures $[22,26,27]$ may become a model system suitable to replace monolayer cultures if these latter type of culturing turns out better to support the growth of MED12-mutated cells.

While the reduced ability of the cells will therefore lead to a re-evaluation of many data obtained in the past it, at the same time, will open novel experimental opportunities and designs. However, the results of the present study show a strong variation in the rate of loss of MED12 mutated cells which at this time has no straightforward explanation like the presence of

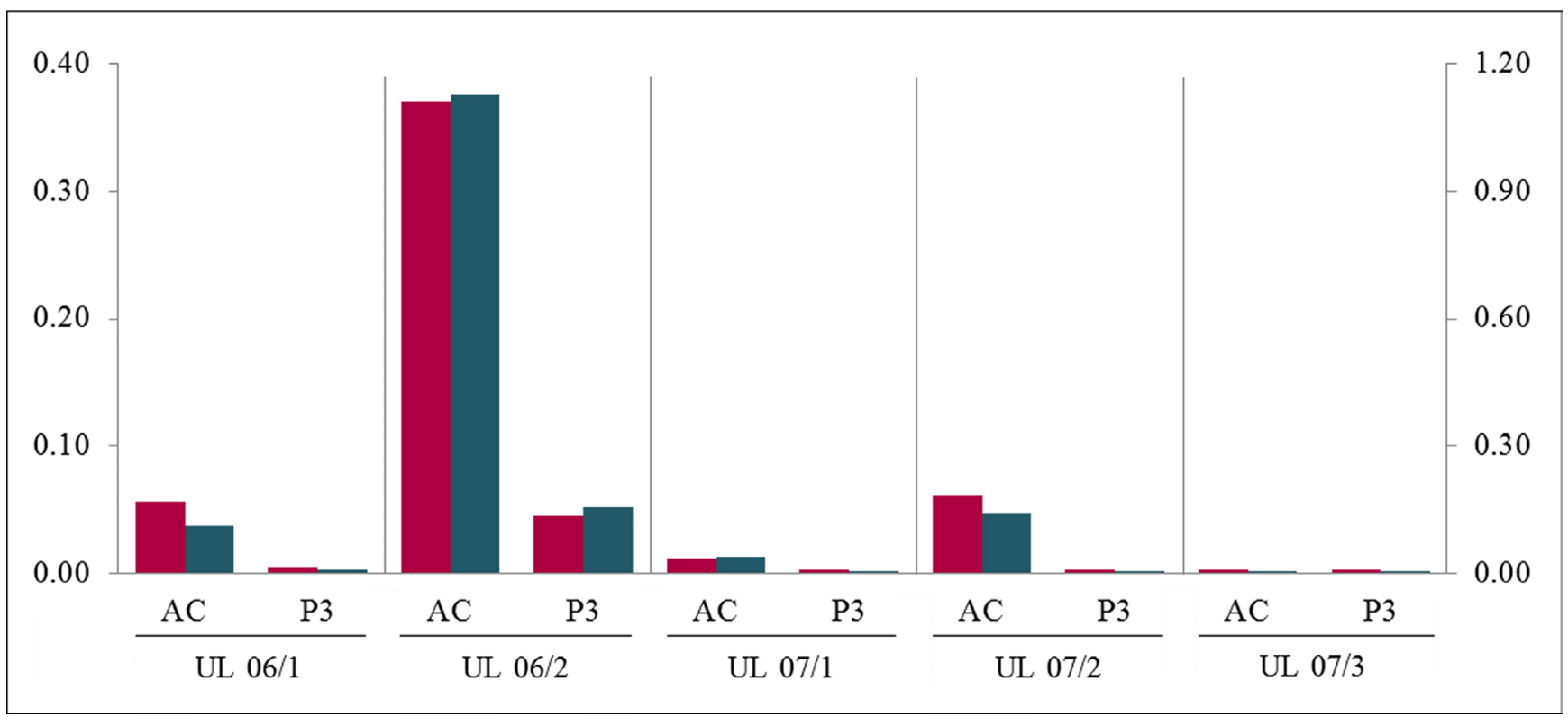

Figure 4: Passaging of cells accelerated the loss of $M E D 12$-mutated cells compared to simple "aging". In all UL the quantification of mutated to non-mutated allele of MED12 mutation shows a decrease of MED12-mutated alleles in passage 3 (P3) compared to the corresponding "aging" primary culture (AC) harvested at the same time period. The quotients of forward sequencing direction (red) were shown on primary axis and of reverse (blue) on secondary axis. 
additional genetic abnormalities as e.g. deletions of the long arm of chromosome 7 or trisomy 12 which are known to accompany MED12 mutations in a considerable percentage of cases. Vice versa, for further experiments it should be determined if there is only an intertumoral variation or if variation can be seen also between different samples from the same tumor. In any case it indicates that investigating large series of samples will be necessary to identify factors contributing to a prolonged survival of MED12-mutated cells in vitro. As to further experiments on larger series it should be noted that obviously not cells from all types of UL do require these factors essential for the growth of the MED12-mutated cells in vitro. UL harboring $H M G A 2$ rearrangements leading to its strong upregulation $[12,20]$ constitute the second largest genetic subgroup of UL the cells of which can survive for many passages under the normal culture conditions $[12,20]$. Also, for further experiments aimed at supplementing various factors, studies on the clonality of the cells should accompany mutation analyses considering recent results obtained on these lesions [28].

\section{MATERIALS AND METHODS}

\section{Tumor samples}

Tumor samples were obtained from hysterectomy specimens immediately after surgery. All samples were taken by an experienced pathologist in order to exclude possible contamination with myometrial tissue. While one piece of each tumor was fixed in paraffin for subsequent histologic examination and one piece was snap frozen in liquid nitrogen, the remaining third was stored in sterile Hank's solution supplemented with antibiotics (500 IU/ml penicillin, $500 \mu \mathrm{g} / \mathrm{ml}$ streptomycin) for cell culturing. Informed written consent was obtained from all patients for genetic characterization of their tumors and use in the present study, respectively, which had been approved by the ethics committee of Ärztekammer Bremen.

\section{Cell cultures}

From tumor samples stored in Hank's solution, cell cultures were set up as described recently [29]. In brief, the tissue samples were minced into small pieces and treated with collagenase (200 U) for 4.5 to $6 \mathrm{~h}$. Dissociated cells were centrifuged and resuspended in culture medium (RPMI 1640 supplemented with 20\% FCS, $100 \mathrm{IU} / \mathrm{ml}$ penicillin and $100 \mu \mathrm{g} / \mathrm{ml}$ streptomycin). The resulting cell suspension of each tumor was transferred into three cell culture flasks and incubated at $37^{\circ} \mathrm{C}$ and $5 \% \mathrm{CO}_{2}$. Cultured cells were enzymatically harvested as "young" primary culture at confluency or passaged using trypsin $(0.05 \%)$ with a $1: 2$ split ratio when reaching $80 \%$ confluency. "Aging" primary cultures were not passaged but continuously supplied with medium after confluency.

\section{DNA isolation}

DNA from fresh frozen tissue samples and cell cultures was isolated using the QIAamp DNA Mini Kit (Qiagen, Hilden, Germany) according to manufacturer's

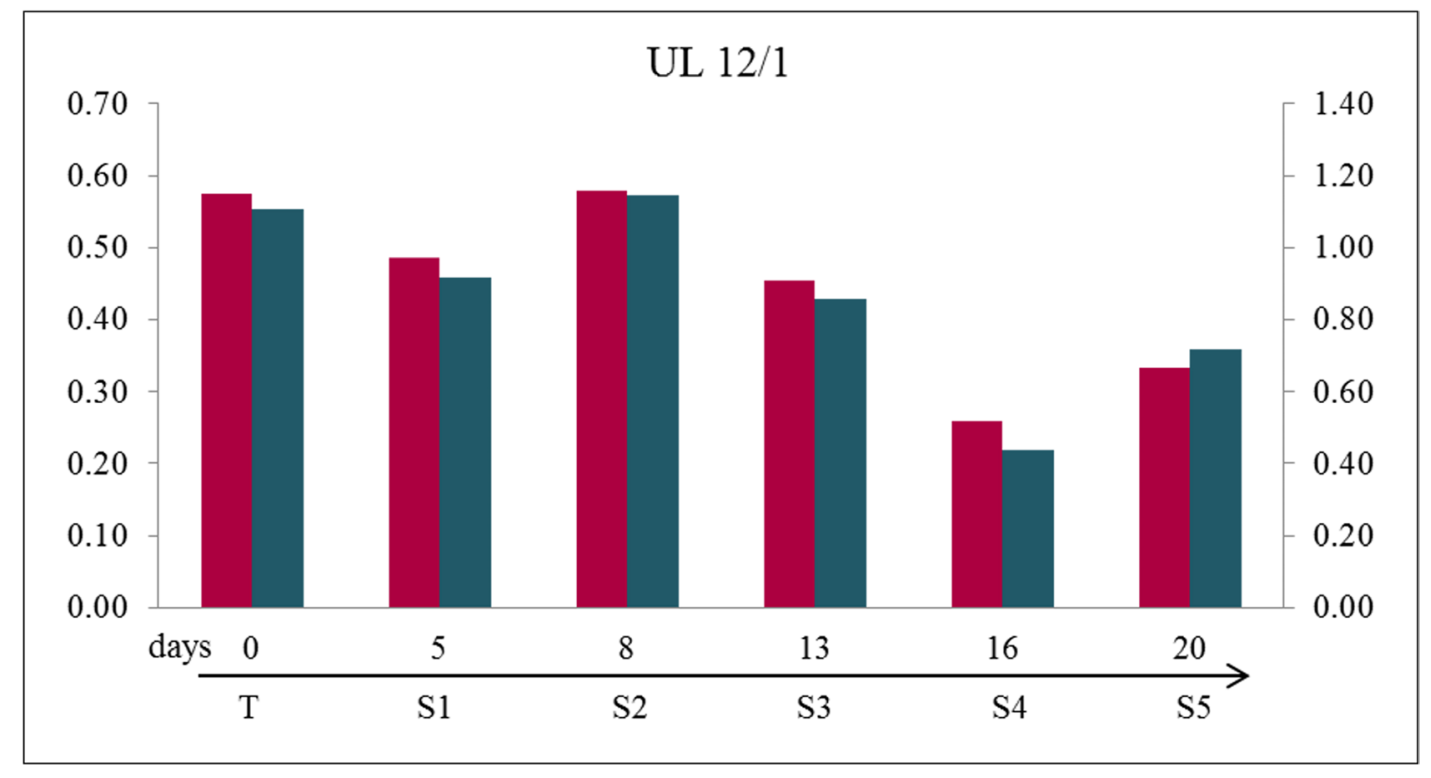

Figure 5: Detachment of cells from the monolayer during "aging" could cause the loss of mutated cells during in vitro culturing. Quantification of mutated to non-mutated allele of MED12 mutation of cells in the supernatant (S1 to S5) revealed a large mutated/non-mutated peak ratio comparable to native tumor tissue (T) indicating a large amount of mutated cells in the supernatant. The numbers represent the days where the supernatants were obtained starting with set up the cell culture using the example of UL 12/1. The quotients of forward sequencing direction (red) were shown on primary axis and of reverse (blue) on secondary axis. 
instructions. Similarly, DNA from cells obtained from the supernatant of cell cultures was processed using the QIAamp DNA Micro Kit (Qiagen). The concentration of genomic DNA was determined by NanoDrop2000 (PeqLab, Erlangen, Germany).

\section{MED12 amplification}

For PCR amplification, 200 to $250 \mathrm{ng}$ of genomic template DNA were used. PCR was performed as follows:
1 cycle at $95^{\circ} \mathrm{C}$ for $15 \mathrm{~min}$ followed by 35 cycles of $94^{\circ} \mathrm{C}$ for $0.5 \mathrm{~min}, 59^{\circ} \mathrm{C}$ for $0.5 \mathrm{~min}, 72^{\circ} \mathrm{C}$ for $0.5 \mathrm{~min}$, and a final extension at $72^{\circ} \mathrm{C}$ for $6 \mathrm{~min}$. Primers used to amplify $M E D 12$ exon2 of the genomic template DNA were 5'-GCCCTTTCACCTTGTTCCTT-3' (forward) and 5'-TGTCCCTATAAGTCTTCCCAACC-3' (reverse), recently described by Mäkinen et al. [3]. Subsequently, PCR-products were electrophoretic separated using a $2 \%$ agarose gel and the desired PCR fragments were extracted by a QIAquick Gel Extraction Kit (Qiagen) according to

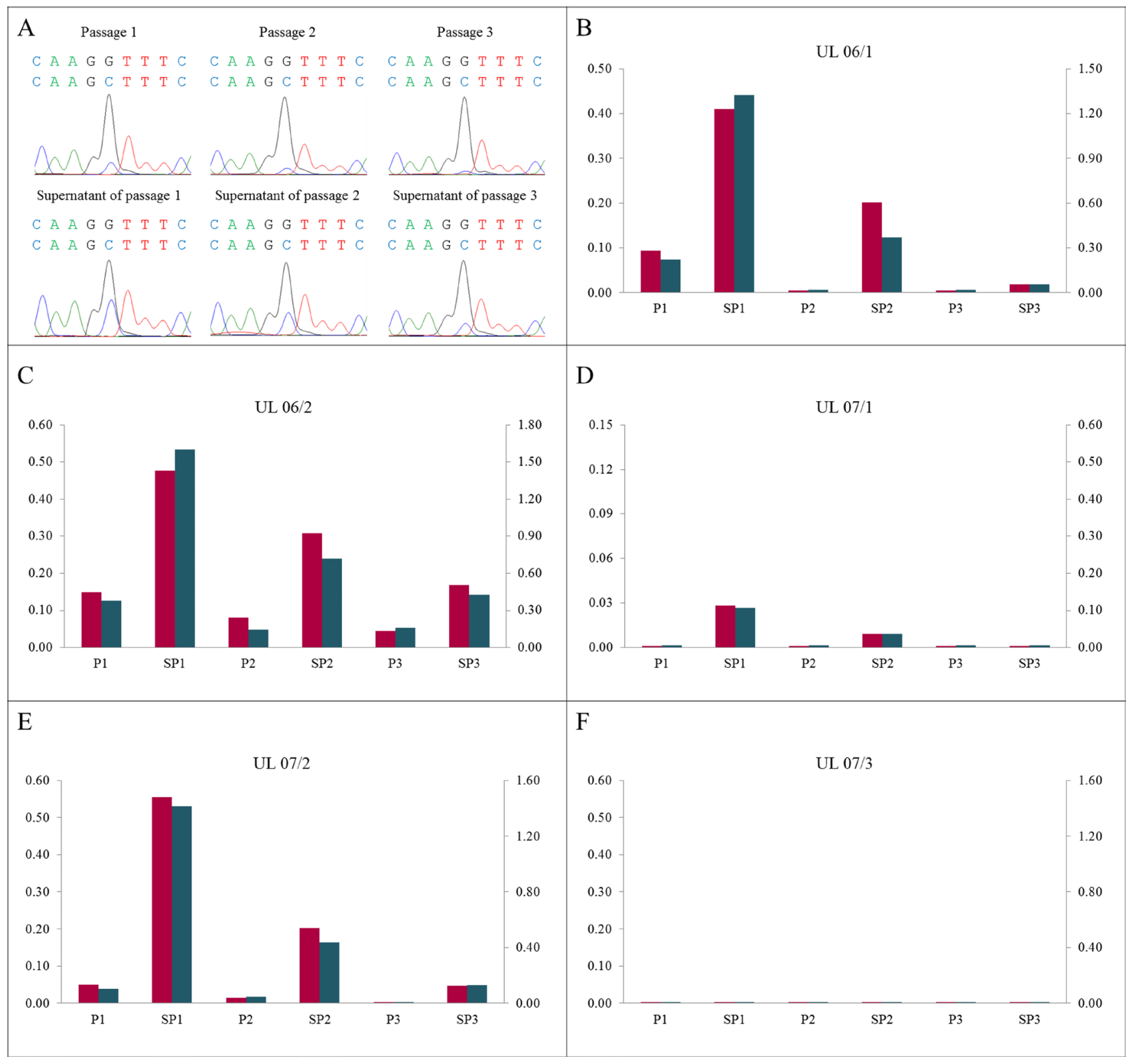

Figure 6: A high proportion of mutated cells in the supernatant of corresponding passages. (A) DNA forward sequences of cells in the supernatants (SP1 to SP3) show a higher "mutated peak" compared to the corresponding passages (P1 to P3) using the example of UL 06/2. (B-F) The mutated/non-mutated peak ratios in the supernatants clearly exceeded the ratios in the corresponding passages in all UL investigated. In increasing numbers of passages and supernatants the ratio decreased. The quotients of forward sequencing direction (red) were shown on primary axis and of reverse (blue) on secondary axis. 
manufacturer's instructions or directly purified using the QIAquick PCR Purification Kit (Qiagen).

\section{Sequencing}

DNA sequencing of the purified PCR products was performed using the GenomeLab DTCS-Quick Start Kit (Beckmann Coulter, Krefeld, Germany) and the GenomeLab GeXP Genetic Analysis System CEQ 8800 (Beckman Coulter) according to manufacturer's instructions. For Sanger sequencing, $20 \mathrm{ng}$ of the PCR product was used as template for both, forward and reverse sequencing. DNA sequencing data were analyzed using the software of Beckman Coulter Instrument (version 10.2) and BioEdit Sequence Alignment Editor (version 7.2.5, Tom Hall, USA). The sequence of $\mathrm{NC}$ 000023.11, $\mathrm{NCBI}$, was used as reference. DNA sequencing data from matching myometria were used as controls.

\section{Quantification of mutated versus wild-type alleles}

In case of MED12 mutation, the electropherogram obtained by Sanger sequencing resulted in two peaks corresponding to the non-mutated and mutated allele at point mutation site in MED12 exon 2. To determine the ratio of mutated versus wild-type alleles, the maximum fluorescence intensity of corresponding peaks was calculated from the respective BioEdit data. The resulting quotients were shown as columns for forward (primary axis) as well as reverse (secondary axis) sequencing direction for each sample. An example is shown in Supplementary Figure 2. In the absence of a detectable mutated peak the ratio was set to 0.001 .

\section{CNV array}

CNV (copy number variation) analysis was performed using premade CytoScan HD Arrays (Affymetrix, Santa Clara, CA) consisting more than 2.4 million markers for copy number alterations and approximately 750,000 markers for single nucleotide polymorphisms (SNPs). Enriched gene coverage results in a marker-base ratio coverage of 1/384 for ISCA, 1/553 for cancer genes, 1/486 for X-chromosomal genes, and $1 / 659$ for 12,000 OMIM genes. The manufacturer's instructions were followed for labelling of $300 \mathrm{ng}$ DNA, and hybridization. After staining and washing using a GeneChip Fluidics Station 450 (Affymetrix) the arrays were scanned by an Affymetrix $30007 \mathrm{G}$ scanner and analyzed through the Affymetrix Chromosome Analysis Suite (ChAS) software (ChAS analysis files for the CytoScan ${ }^{\circledR}$ HD Array version NA33). Numbering of map positions was based on hg19 (NCBI Build 37 reference sequence). Filter settings of copy number changes across the genome were $\geq 25 \mathrm{kbp}$ and marker count $\geq 30$.

\section{ACKNOWLEDGMENTS}

We wish to thank Heike Janecek-Meyer and Karin Zielke for their excellent technical assistance and Dominique Markowski for the primers.

\section{CONFLICTS OF INTEREST}

None.

\section{REFERENCES}

1. Cardozo ER, Clark AD, Banks NK, Henne MB, Stegmann BJ, Segars JH. The estimated annual cost of uterine leiomyomata in the United States. Am J Obstet Gynecol. 2012; 206:211.e1-9. doi: 10.1016/j.ajog.2011.12.002.

2. Segars JH, Akopians AL. The two health disparities of uterine fibroids. Fertil Steril. 2013; 99:1851-2. doi: 10.1016/j.fertnstert.2013.03.002.

3. Mäkinen N, Mehine M, Tolvanen J, Kaasinen E, Li Y, Lehtonen HJ, Gentile M, Yan J, Enge M, Taipale M, Aavikko M, Katainen R, Virolainen E, et al. MED12, the mediator complex subunit 12 gene, is mutated at high frequency in uterine leiomyomas. Science. 2011; 334:2525. doi: 10.1126/science.1208930.

4. Markowski DN, Bartnitzke S, Löning T, Drieschner N, Helmke BM, Bullerdiek J. MED12 mutations in uterine fibroids - their relationship to cytogenetic subgroups. Int $\mathrm{J}$ Cancer. 2012; 131:1528-36. doi: 10.1002/ijc.27424.

5. McGuire MM, Yatsenko A, Hoffner L, Jones M, Surti U, Rajkovic A. Whole exome sequencing in a random sample of North American women with leiomyomas identifies MED12 mutations in majority of uterine leiomyomas. PLoS One. 2012; 7:e33251. doi: 10.1371/journal.pone.0033251.

6. Bertsch E, Qiang W, Zhang Q, Espona-Fiedler M, Druschitz S, Liu Y, Mittal K, Kong B, Kurita T, Wei JJ. MED12 and HMGA2 mutations: two independent genetic events in uterine leiomyoma and leiomyosarcoma. Mod Pathol. 2014; 27:1144-53. doi: 10.1038/modpathol.2013.243.

7. Heinonen HR, Sarvilinna NS, Sjöberg J, Kämpjärvi K, Pitkänen E, Vahteristo $\mathrm{P}$, Mäkinen N, Aaltonen LA. MED12 mutation frequency in unselected sporadic uterine leiomyomas. Fertil Steril. 2014; 102:1137-42. doi: 10.1016/j.fertnstert.2014.06.040.

8. Clark AD, Oldenbroek M, Boyer TG. Mediator kinase module and human tumorigenesis. Crit Rev Biochem Mol Biol. 2015; 50:393-426. doi: 10.3109/10409238.2015.1064854.

9. Zaitseva M. In vitro culture significantly alters gene expression profiles and reduces differences between myometrial and fibroid smooth muscle cells. Mol Hum Reprod. 2006; 12:187-207. doi: 10.1093/molehr/gal018.

10. Chang B, Myatt L, Cui XL. Loss of proliferative capacity in a retroviral immortalized human uterine smooth muscle 
cell line derived from leiomyoma is restored by hTERT overexpression. Reprod Sci. 2009; 16:1062-71. doi: 10.1177/1933719109344773.

11. Suzuki K, Nakabayashi K, Yamada AY, Lodhi RSZ, Hazama R, Ebina Y, Yamada H. Recombinant H2 relaxin inhibits apoptosis and induces cell proliferation in cultured leiomyoma cells without affecting those in cultured normal myometrial cells. Fertil Steril. 2012; 97:734-41. doi: 10.1016/j.fertnstert.2011.12.011.

12. Markowski DN, Tadayyon M, Bartnitzke S, Belge G, Maria Helmke B, Bullerdiek J. Cell cultures in uterine leiomyomas: Rapid disappearance of cells carrying MED12 mutations. Genes Chromosomes Cancer. 2014; 53:317-23. doi: $10.1002 /$ gcc. 22142 .

13. Donnez J, Tomaszewski J, Vázquez F, Bouchard $\mathrm{P}$, Lemieszczuk B, Baró F, Nouri K, Selvaggi L, Sodowski K, Bestel E, Terrill P, Osterloh I, Loumaye E. Ulipristal Acetate versus Leuprolide Acetate for Uterine Fibroids. N Engl J Med. 2012; 366:421-32. doi: 10.1056/NEJMoa1103180.

14. Donnez J, Tatarchuk TF, Bouchard P, Puscasiu L, Zakharenko NF, Ivanova T, Ugocsai G, Mara M, Jilla MP, Bestel E, Terrill P, Osterloh I, Loumaye E. Ulipristal acetate versus placebo for fibroid treatment before surgery. N Engl J Med. 2012; 366:409-20. doi: 10.1056/NEJMoa1103182.

15. Ohara N. Action of progesterone receptor modulators on uterine leiomyomas. Clin Exp Obstet Gynecol. 2008; 35:165-6. Available from http://www.ncbi.nlm.nih.gov/ pubmed/18754282

16. Roeder H, Jayes F, Feng L, Leppert PC. CDB-4124 does not cause apoptosis in cultured fibroid cells. Reprod Sci. 2011; 18:850-7. doi: 10.1177/1933719111399929.

17. Zhu Y, Zhang T, Xie S, Tu R, Cao Y, Guo X, Zhou J, Zhou X, Cao L. Gestrinone inhibits growth of human uterine leiomyoma may relate to activity regulation of ER $\alpha$, Src and P38 MAPK. Biomed Pharmacother. 2012; 66:56977. doi: 10.1016/j.biopha.2012.02.003.

18. Segars JH, Parrott EC, Nagel JD, Guo XC, Gao X, Birnbaum LS, Pinn VW, Dixon D. Proceedings from the Third National Institutes of Health International Congress on Advances in Uterine Leiomyoma Research: comprehensive review, conference summary and future recommendations. Hum Reprod Update. 2014; 20:309-33. doi: 10.1093/ humupd/dmt058.

19. Gross KL, Neskey DM, Manchanda N, Weremowicz S, Kleinman MS, Nowak RA, Ligon AH, Rogalla P, Drechsler K, Bullerdiek J, Morton CC. HMGA2 expression in uterine leiomyomata and myometrium: quantitative analysis and tissue culture studies. Genes Chromosomes Cancer. 2003; 38:68-79. doi: 10.1002/gcc.10240.

20. Holzmann C, Markowski DN, Bartnitzke S, Koczan D, Helmke BM, Bullerdiek J. A rare coincidence of different types of driver mutations among uterine leiomyomas (UL). Mol Cytogenet. 2015; 8:76-82. doi: 10.1186/s13039-0150177-9.
21. Severino MF, Murray MJ, Brandon DD, Clinton GM, Burry KA, Novy MJ. Rapid loss of oestrogen and progesterone receptors in human leiomyoma and myometrial explant cultures. Mol Hum Reprod. 1996; 2:823-8.

22. Malik M, Britten J, Cox J, Patel A, Catherino WH. Gonadotropin-releasing hormone analogues inhibit leiomyoma extracellular matrix despite presence of gonadal hormones. Fertil Steril. 2015; 105:214-24. doi: 10.1016/j. fertnstert.2015.09.006.

23. Aissani B, Zhang K, Wiener H. Follow-up to genomewide linkage and admixture mapping studies implicates components of the extracellular matrix in susceptibility to and size of uterine fibroids. Fertil Steril. 2015; 103:528-34. e13. doi: 10.1016/j.fertnstert.2014.10.025.

24. Lemeer S, Gholami AM, Wu Z, Kuster B. Quantitative proteome profiling of human myoma and myometrium tissue reveals kinase expression signatures with potential for therapeutic intervention. Proteomics. 2015; 15:356-64. doi: 10.1002/pmic.201400213.

25. Islam MS, Protic O, Ciavattini A, Giannubilo SR, Tranquilli AL, Catherino WH, Castellucci M, Ciarmela P. Tranilast, an orally active antiallergic compound, inhibits extracellular matrix production in human uterine leiomyoma and myometrial cells. Fertil Steril. 2014; 102:597-606. doi: 10.1016/j.fertnstert.2014.05.013.

26. Malik M, Catherino WH. Development and validation of a three-dimensional in vitro model for uterine leiomyoma and patient-matched myometrium. Fertil Steril. 2012; 97:128793. doi: 10.1016/j.fertnstert.2012.02.037.

27. Levy G, Malik M, Britten J, Gilden M, Segars J, Catherino WH. Liarozole inhibits transforming growth factor- $\beta 3$ mediated extracellular matrix formation in human threedimensional leiomyoma cultures. Fertil Steril. 2014; 102:272-281.e2. doi: 10.1016/j.fertnstert.2014.03.042.

28. Holdsworth-Carson SJ, Zaitseva M, Vollenhoven BJ, Rogers PAW. Clonality of smooth muscle and fibroblast cell populations isolated from human fibroid and myometrial tissues. Mol Hum Reprod. 2014; 20:250-9. doi: 10.1093/ molehr/gat083.

29. Klemke M, Meyer A, Nezhad MH, Bartnitzke S, Drieschner N, Frantzen C, Schmidt EH, Belge G, Bullerdiek J. Overexpression of HMGA2 in uterine leiomyomas points to its general role for the pathogenesis of the disease. Genes Chromosomes Cancer. 2009; 48:171-8. doi: $10.1002 /$ gcc. 20627 . 